PROCEEDINGS OF THE

AMERICAN MATHEMATICAL SOCIETY

Volume 131, Number 9, Pages 2633-2638

S 0002-9939(03)07138-7

Article electronically published on April 23, 2003

\title{
FORMALITY OF EQUIVARIANT INTERSECTION COHOMOLOGY OF ALGEBRAIC VARIETIES
}

\author{
ANDRZEJ WEBER
}

(Communicated by Mohan Ramachandran)

\begin{abstract}
We present a proof that the equivariant intersection cohomology of any complete algebraic variety acted by a connected algebraic group $G$ is a free module over $H^{*}(B G)$.
\end{abstract}

\section{INTRODUCTION}

Let $X$ be a complex algebraic variety, and let $G$ be a linear algebraic group acting algebraically on $X$. Denote by $I H_{G}^{*}(X)=H^{*}\left(E G \times_{G} X ; \mathcal{I C} \mathcal{C}_{G}\right)$ the equivariant intersection cohomology of $X$ with rational coefficients, first introduced in $[\mathrm{Br}]$. It is a module over $H^{*}(B G)$. We say that $X$ is formal if $I H_{G}^{*}(X)$ is free. We have a spectral sequence

$$
E_{2}^{p q}=H^{p}\left(B G, I H^{q}(X)\right) \Rightarrow I H_{G}^{p+q}(X) .
$$

Suppose that $G$ is connected. Then $B G$ is 1 -connected and the coefficients are not twisted. Then the spectral sequence $\left(^{*}\right)$ degenerates if and only if $X$ is formal.

The aim of this note is to show that complete $G$-varieties are formal. We will prove:

Theorem 1. Let $X$ be a complete algebraic variety acted by a connected algebraic linear group. Then $X$ is formal.

In this way we add to the list of GKM, 14.1] an important class of examples. In fact Theorem 1 is known to specialists. Each of the proofs comes back to the fundamental paper of Beilinson-Bernstein-Deligne-Gabber, [BBD]. After all, in the most general case, Theorem 1 follows from a simple weight argument, as presented in Remark 7. Nevertheless, we think it is worth comparing several approaches to equivariant intersection cohomology and methods of possible proofs.

I would like to thank Prof. Andrzej Białynicki-Birula for giving me a hint in a crucial point. I thank Prof. Pierre Deligne for valuable remarks and for assuring me that the methods of [BBD] work for algebraic spaces. I thank Prof. Frances Kirwan for her comments. I would like also to thank Marcin Chałupnik for help during the preparation of this note.

Received by the editors February 8, 2001.

2000 Mathematics Subject Classification. Primary 14F43, 55N25; Secondary 55N33.

Key words and phrases. Complete algebraic varieties, equivariant intersection cohomology.

This research was partially supported by grant KBN 2P03A 00218 and by the European Commission RTN Geometric Analysis. 


\section{Proof For PRojective VARIETIES}

Our proof of Theorem 1 uses the decomposition theorem or reduction to a finite characteristic, which are strong tools. Thus we think that it is worth noting that for projective $X$ the situation is much simpler. We proceed as follows:

Step 1: We choose an equivariant embedding of $X$ into a projective space. This is possible by a result of Sumihiro [Sum, Theorem 1].

Step 2: Let $h \in H^{2}(X)$ be the class of the hyperplane section. We show that $h$ can be lifted to $H_{G}^{2}(X)$. This is so for $X=\mathbb{P}^{N}$ and $G=G L(N+1)$ acting naturally. Indeed, take an infinite Grassmannian as a model of $B G$. Then

$$
E G \times{ }_{G} \mathbb{P}^{N}=\left\{(V, l): l \subset V \subset \mathbb{C}^{\infty}, \operatorname{dim} l=1, \operatorname{dim} V=N+1\right\} .
$$

One has a tautological bundle, whose Chern class is a lift of $-h$. If $X \subset \mathbb{P}^{N}$ and $G$ acts linearly, we construct a lift for $\mathbb{P}^{N}$ by naturality with respect to $G$ and we restrict it to $X$.

Step 3: We have a map of spectral sequences

$$
L: E_{r}^{p, q} \rightarrow E_{r}^{p, q+2},
$$

which is induced by the multiplication by a cycle (or a form) representing $h$. Let $d=\operatorname{dim} X$. By the hard Lefschetz theorem

$$
L^{k}: E_{2}^{p, d-k}=H^{p}(B G) \otimes I H^{d-k}(X) \rightarrow E_{2}^{p, d+k}=H^{p}(B G) \otimes I H^{d+k}(X)
$$

is an isomorphism for every $p$ and $k$. From the Lefschetz criterion, [D1], it follows that the spectral sequence degenerates, as noticed in [ $\mathrm{Br}, 4.2 .2]$.

This argument works for varieties over any field of any characteristic. One should replace the ordinary cohomology and intersection cohomology by their étale version. In Step 3 an isomorphism $I H_{G}^{*}(X) \simeq H^{*}(B G) \otimes I H^{*}(X)$ can be made canonical by [D3]. It would depend only on the embedding $X \subset \mathbb{P}^{N}$ and the representation $G \rightarrow G L(k)$.

For smooth $X$ Step 2 can also be carried out by methods of symplectic geometry. Let $K$ be a maximal compact subgroup of $G$. Then $H_{G}^{*}\left(\mathbb{P}^{N}\right) \simeq H_{K}^{*}\left(\mathbb{P}^{N}\right)$. The class $h$ is represented by a $K$-equivariant symplectic form $\omega$. One can define a moment map by an explicit formula [K, 2.5]. The choice of the moment map is precisely a lift of $\omega$ to $H_{K}^{2}\left(\mathbb{P}^{N}\right)$ in the Cartan model of $\Omega^{*}\left(E K \times_{K} \mathbb{P}^{N}\right)$; see [AB. 6.18]. Another proof for smooth projective varieties can be obtained by means of a Morse function coming from a moment map; see $[\mathrm{K}, 5.8]$.

In the rest of the paper we will show how to deduce formality for an arbitrary complete variety, not necessarily projective.

\section{Algebraic models of $E G \times_{G} X$}

There are several constructions of $B G$ that can be performed in the category of algebraic varieties. Of course $B G$ itself cannot be an algebraic variety, since it cannot be of finite dimension. There are two well-known approaches of giving a meaning to $B G$ : an approximation or the simplicial construction.

The first approach is to approximate $B G$ by algebraic varieties $B_{n} G$. For a detailed exposition see [T]. If $G \subset G L(k)$, then for example we take as $E_{n} G$ the Stiefel variety of $k$ independent vectors in $\mathbb{C}^{n+k}$. The quotient $B_{n} G=E_{n} G / G$ 
exists and it is an algebraic variety. It can be presented as the quotient of groups $G L(n+k) / H$, where $H=\left(\begin{array}{cc}G & * \\ 0 & G L(n)\end{array}\right)$. Another candidate for an approximation is the quotient $B_{n}^{\prime} G=G L(n+k) / H^{\prime}$, where $H^{\prime}=\left(\begin{array}{cc}G & 0 \\ 0 & G L(n)\end{array}\right) \simeq G \times G L(n)$. In this case

where $H_{0}^{\prime}=\left(\begin{array}{ll}I & 0 \\ 0 & G L(n)\end{array}\right)$.

$$
E_{n}^{\prime} G=G L(n+k) / H_{0}^{\prime},
$$

Now we construct the associated bundle. Using the approximation $B_{n}^{\prime} G$ we have $E_{n}^{\prime} G \times_{G} X=G L(n+k) \times_{H^{\prime}} X$ (where $G L(n)$ acts trivially on $\left.X\right)$. But even here, with such a simple construction, the resulting space does not have to be a variety. The spaces $E_{n}^{\prime} G \times_{G} X$ (approximations of the Borel construction) are quotients of $E_{n}^{\prime} G \times X$ by $G$ and it can be shown that they are algebraic spaces. Let us quote B-B:

Theorem 2. Let $G$ be an algebraic group. Let $E$ and $X$ be algebraic spaces and let $E$ be a principal locally isotrivial $G$-fibration. Assume that $E$ is normal. Then $E \times_{G} X$ exists in the category of algebraic spaces. If, moreover, $E$ is an algebraic variety and $X$ is normal and can be covered by $G$-invariant open quasi-projective subsets, then $E \times_{G} X$ is an algebraic variety.

Note that, by [B-B Theorem 3], if $H$ is a connected affine algebraic group, $G \subset H$ and $X$ normal, then $H \times_{G} X$ is an algebraic variety if and only if the assumption about covers is fulfilled. For the proof of formality one would like to apply the decomposition theorem (or other methods based on Weil conjectures). It is clear that either we have to make some assumptions about $X$, or to generalize the results of $[\mathrm{BBD}]$ to the case of algebraic spaces.

In order to avoid algebraic spaces it is possible to apply a simplicial construction of $B G$; see [D2, 6.1]. Then $(B G)$. is a simplicial variety: $(B G)_{n}=G^{n}$. The associated Borel construction is also a simplicial variety: $\left(E G \times_{G} X\right)_{n}=G^{n} \times X$. The topological realization of the Borel construction coincides with the realization of the quotient stack $X / G$ as defined in [Si, §8].

Remark 3. Both constructions of $B G$ are compared in VoM Lemma 4.1.18 and Prop. 4.2.4]. Applying any of the described constructions we obtain a Hodge structure on $H^{*}(B G)$ which is pure; see [D2, 9.1]. If we perform the same constructions for groups over a finite field, we also obtain a pure structure with respect to the action of the Frobenius automorphism.

Remark 4. Suppose the base field is $\mathbb{F}_{q}$. Then the action of the Frobenius automorphism $F r^{q}: x \mapsto x^{q}$ induces an operation on $H_{e t}^{*}\left(B G ; \mathbb{Q}_{\ell}\right)$, which is just the Adams operation $\psi^{q}$; compare [AM] and [Sul, Ch. 5, §4].

\section{Degeneration of the spectral sequence WITHOUT THE ASSUMPTION OF PROJECTIVITY}

Suppose $X$ is a complete algebraic variety. We replace $X$ by its normalization. It does not change the intersection cohomology. Assume that $X$ can be covered by $G$ invariant open quasi-projective subsets. Then the space $E_{n} G \times_{G} X$ (or $E_{n}^{\prime} G \times_{G} X$ ) is an algebraic variety by Theorem 2 . The projection

$$
E_{n} G \times{ }_{G} X \rightarrow B_{n} G
$$

is a proper algebraic morphism. 
Corollary 5. If the normalization of $X$ can be covered by $G$-invariant quasiprojective open subsets, then the spectral sequence (*) converging to $I H^{*}\left(E_{n} G \times_{G} X\right)$ degenerates at the $E_{2}$ term.

Proof. The corollary follows from the decomposition theorem of [BBD].

Remark 6 . We would be able to remove the assumption about covers, provided that we proved the decomposition theorem for separable algebraic spaces. The statement of [BBD, 6.2.5] seems to remain valid. To prove it one would have to develop the formalism of mixed sheaves for algebraic spaces over finite fields.

\section{The End of The PROOF}

If $X$ can be covered by $G$-invariant quasiprojective open subsets, then $E G \times_{G} X$ can be approximated by algebraic varieties. The formality follows from Corollary 5 . For arbitrary complete $X$ there is a way of going around the problem with $E G \times_{G} X$ not being a variety. First we consider the maximal torus $T \subset G$. The classifying space for the torus can be realized as a product of infinite projective spaces. Then the approximation of $E T \rightarrow B T$ is locally Zariski trivial. Thus $E T \times_{T} X$ is a limit of algebraic varieties. We can directly apply the techniques of [BBD] to deduce that the spectral sequence $(*)$ degenerates for $T$. The restriction to the maximal torus induces an injection $H^{*}(B G) \hookrightarrow H^{*}(B T)$ and an injection of the $E_{2}$ terms of the spectral sequences $(*)$. Therefore, since the differentials vanish in the $E_{2}$ term for $T$, they vanish in the $E_{2}$ term for $G$. Then $E_{2}=E_{3}$ and we continue our reasoning. We find that all the differentials in the spectral sequence for $G$ vanish. The reduction to the maximal torus was described in [Gi, §4, proof of 3.3].

Remark 7. There is another solution to the problem with Borel construction. We apply the simplicial model of $E G$. We reduce the base ring to a finite field. Now we follow Deligne's suggestion: the Galois group acts on the spectral sequence $\left.{ }^{*}\right)$. Since $H^{*}(B G)$ and $I H^{*}(X)$ are pure, $E_{2}^{p q}$ is pure of weight $p+q$. All the differentials in the spectral sequence vanish because they mix weights. Note that this proof works for noncomplete algebraic varieties, provided that $I H^{*}(X)$ is pure. One can generalize our proof further and consider cohomology with coefficients in an arbitrary 'sheaf' $\mathcal{F}$, an object of the derived category. In this case a suitable extension of $\mathcal{F}$ on the simplicial variety $\left(E G \times_{G} X\right)$. has to be chosen to make sense of the equivariant cohomology. The extension $\mathcal{F}_{G}$ should be an equivariant sheaf in the sense of [BL] and it should be defined over a finitely generated subring $R \subset \mathbb{C}$. Moreover $\mathcal{F}_{G}$ should have enough good reductions to finite fields, so that

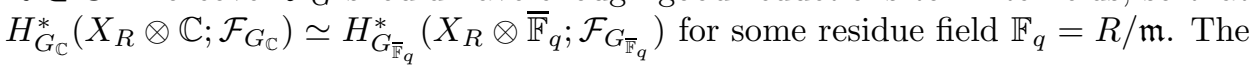
conclusion is the same: purity of $H^{*}(X ; \mathcal{F})$ implies formality.

\section{Algebra structure}

Theorem 1 shows that the $H^{*}(B G)$-module structure of $H_{G}^{*}(X)$ carries no information about the action. The algebra structure of $H_{G}^{*}(X)$ is more interesting, but hard to recover. It involves some characteristic classes of the action.

Example 8. Let $X=\mathbb{P}^{N}$ and let $G=G L(N+1)$ with the obvious action on $X$ (as in Step 2 of $\S 1$ ). The effect of the Borel construction is the projectivization of the tautological bundle over $B G$. The cohomology algebra is well known:

$$
H_{G}^{*}(X)=\mathbb{Q}\left[c_{1}, c_{2}, \ldots, c_{N+1}, h\right] /\left(c_{N+1}-c_{N} h+\cdots+c_{1}(-h)^{N}+(-h)^{N+1}\right) .
$$




\section{DuAl structure}

Due to Koszul duality GKM], [MW], the nonequivariant cohomology of $X$ is equipped with the structure of a module over $H_{*}(G)$ : for $\lambda \in H_{k}(G)$ and $x \in H^{l}(X)$ we set $\lambda \cdot x=\lambda \backslash \mu^{*}(x) \in H^{l-k}(X)$, where $\mu: G \times X \rightarrow X$ is the action and $\backslash$ is the slant product $\backslash: H_{k}(G) \otimes H^{l}(G \times X) \rightarrow H^{l-k}(X)$. According to [GKM] 9.3] if the spectral sequence $\left(^{*}\right)$ degenerates, then the cohomology of $X$ is a trivial module over $H_{*}(G)$, even on the level of the derived category of $H_{*}(G)$-modules. Therefore for any $\lambda$ with $\operatorname{deg} \lambda>0$ we have $\lambda \cdot x=0$, i.e.:

Corollary 9. The structure of an $H_{>0}(G)$-module on $I H^{*}(X)$ is trivial for a complete variety $X$ acted by a connected group $G$.

This generalizes a result of Deligne [D2, 9.1.7].

\section{REFERENCES}

[AB] Atiyah, M.F.; Bott, R., The moment map and equivariant cohomology, Topology 23 (1984), 1-28. MR 85e:58041

[AM] Adams, J. F.; Mahmud, Z., Maps between classifying spaces, Inv. Math. 35 (1976), 1-41. MR 54:11331

[BBD] Beilinson, A.A.; Bernstein, J.; Deligne, P., Faisceaux pervers, Asterisque 100 (1982). MR 86g:32015

[BL] Bernstein, J.; Lunts, V., Equivariant sheaves and functors, Lecture Notes in Mathematics, vol. 1578, Springer-Verlag, Berlin, 1994. MR 95k:55012

[B-B] Białynicki-Birula A., On induced actions of algebraic groups, Ann. Inst. Fourier, Grenoble 43 (2) (1993), 365-368. MR 94c:14041

[Br] Brylinski, J-L., Equivariant intersection cohomology, Kazhdan-Lusztig theory and related topics. Proceedings of an AMS special session, held May 19-20, 1989 at the University of Chicago, Lake Shore Campus, Chicago, IL, USA. American Mathematical Society. Contemp. Math. 139 (Deodhar, Vinay, eds.), Providence, RI:, 1992, pp. 5-32. MR 94c:55010

[D1] Deligne, P., Theoreme de Lefschetz et criteres de degenerescence de suites spectrales, Publ. Math., Inst. Hautes Etud. Sci. 35 (1968), 107-126. MR 39:5582

[D2] Deligne, P., Theorie de Hodge. III, Publ. Math., Inst. Hautes Etud. Sci. 44 (1974), 5-77. MR 58:16653b

[D3] Deligne, P., Decompositions dans la categorie derivee, Motives. Proceedings of the summer research conference on motives, held at the University of Washington, Seattle, WA, USA, July 20-August 2, 1991. American Mathematical Society. Proc. Symp. Pure Math. 55 (Jannsen, U. et al., eds.), Providence, RI: 1994, pp. 115-128. MR 95h:18013

[Gi] Ginzburg, V. A., Equivariant cohomology and Kahler geometry (Russian), Funktsional. Anal. i Prilozhen. 21 (4), 19-34. MR 89b:58013

[GKM] Goresky, M.; Kottwitz, R.; MacPherson, R., Equivariant cohomology, Koszul duality, and the localization theorem, Invent. Math. 131 (1) (1998), 25-83. MR 99c:55009]

[K] Kirwan, F. C., Cohomology of quotients in symplectic and algebraic geometry, Mathematical Notes, vol. 31, Princeton, New Jersey: Princeton University Press, 1984. MR 86i: 58050

[MW] Maszczyk, T.; Weber, A., Koszul duality for modules over Lie algebra, Duke Math. J. 112 (2002), no. 3, 511-520

[VoM] Morel, F.; Voevodsky, V., A'-homotopy theory of schemes, Preprint 1998. http: //www.math.uiuc.edu/K-theory

[Sul] Sullivan, D., Geometric topology. Part I. Localization, periodicity, and Galois symmetry, Massachusetts Institute of Technology, Cambridge, Mass., 1971. MR 58:13006a

[Si] Simpson, C., The topological realization of a simplicial presheaf, q-alg/9609004.

[Sum] Sumihiro H., Equivariant completions I, Math. Kyoto Univ. 14 (1974), 1-28. MR 49:2732 
[T] Totaro, B., The Chow ring of a classifying space, math.AG/9802097; Algebraic $K$-theory. Proceedings of an AMS-IMS-SIAM summer research conference, Seattle, WA, USA, July 13-24 1997 Proc. Symp. Pure Math. Vol 67 (Raskind, Wayne et al., eds.), Providence, RI: American Mathematical Society. Proc., 1999, pp. 249-281. MR 2001f:14011

Institute of Mathematics, Warsaw University, Ul. Banacha 2, 02-097 Warszawa, POLAND

E-mail address: aweber@mimuw.edu.pl 